\title{
Execution of Password Authentication Encrypted Negative Password using MD5
}

\author{
$\mathrm{Mr}^{1}$ M.Sridharan B.Tech, Mrs. ${ }^{2}$ L.Karthika. M.E, \\ ${ }^{1}$ Student M.E CSE Dept, ${ }^{2}$ Assistant professor Dept of Computer science and Engineering, \\ Gojan School of Business and Technology, Redhills, Chennai.
}

\begin{abstract}
Hypothetical Secure mystery express accumulating is a crucial perspective in systems considering secret expression affirmation, which is at this point the most extensively used approval method, disregarding its some security abandons. In this paper, we propose a mysterious key affirmation structure that is expected for secure mystery key storing and could be adequately organized into existing approval systems. In our construction, first, they got plain mystery word from a client is worked out a cryptographic hash work (e.g., SHA-256). By then, the hashed secret expression is changed over into a negative mystery state. Finally, the negative mystery key is encoded into an Encrypted Negative Password (contracted as ENP) using a symmetric-key computation (e.g., AES), and multi activity encryption could be used to furthermore improve security. The cryptographic hash work and symmetric encryption make it difficult to break passwords from ENPs. Furthermore, there are lots of relating ENPs for ensured plain mystery word, which makes pre-computation attacks (e.g., question table attack and rainbow table attack) infeasible. The count multifaceted nature examinations and relationships show that the ENP could go against inquiry table attack and give more grounded secret word security under word reference attack. It merits referring to that the ENP doesn't present extra segments (e.g., salt); other than this, the ENP could regardless face precomputation attacks. Most importantly, the ENP is the primary mystery state confirmation plot that joins the cryptographic hash work, the negative mystery key and the symmetric-key computation, without the necessity for additional information.
\end{abstract}

Keywords - Plain password, Cryptographic hash function, ENP, Symmetric key algorithm.

\section{INTRODUCTION}

(Planning and Implementation of Authentication Scheme by Encrypted Negative Password)

Inferable from the improvement of the Internet, countless online administrations have arisen, in which secret word verification is the most broadly utilized confirmation procedure, for it is accessible requiring little to no effort and simple to convey Subsequently, secret key security consistently pulls in extraordinary interest from the scholarly community and industry Notwithstanding extraordinary examination accomplishments on secret key security, passwords are as yet broken since clients' imprudent practices. For example, numerous clients regularly select powerless passwords they will in general reuse same passwords in various frameworks they typically set their passwords utilizing recognizable jargon for its benefit to recollect in expansion, framework issues may cause secret key settles. It is extremely hard to get passwords from high security frameworks. From one perspective, taking verification information tables (containing usernames and passwords) in high security frameworks is troublesome. On the other hand, when completing a web based speculating assault, there is normally a cut-off to the quantity of login endeavours. Be that as it may, passwords might be spilled from frail frameworks. Weaknesses are continually being found, and not everything frameworks could be opportune fixed to oppose assaults, which offers enemies a chance to illicitly get to frail frameworks. Truth be told, some old frameworks are more defenceless due to their absence of support. At long last, since passwords are regularly reused, foes may sign into high security frameworks through broke passwords from frameworks of low security.

\section{BACKGROUND}

Joseph Bonneau, Cormac Harley, said that the hypothesis on passwords has fall behind training, where enormous suppliers use back-end smarts to get by with blemished innovation. Oversimplified models of client and assailant 
practices have driven the exploration local area to accentuate some unacceptable dangers. Validation is an arrangement issue manageable to AI, with numerous signs notwithstanding the secret word accessible to large Web administrations. Passwords will proceed as a valuable sign for a long time to come, where the objective isn't invulnerable security however lessening hurt at adequate expense. Ch Gopal Krishna and R BalaDinakar, This improvement brings extraordinary accommodation yet additionally builds the likelihood of presenting passwords to bear surfing assaults. Aggressors can notice straightforwardly or utilize outside recording gadgets to gather clients' certifications. To survive this precarious, we proposed a novel confirmation framework Pass Grid, in light of graphical passwords to oppose shoulder riding assaults. With a one-time legitimate login pointer and circulate level and vertical bars covering the whole extent of pass-pictures, Pass Matrix offers no clue for aggressors to sort out or limited down the secret phrase even they lead numerous camera-based assaults. Mr. RudreshGurav, Ms. Leena Dabhade, To increment secret word security, online confirmation frameworks have begun to uphold stricter secret phrase approaches. We present another measurement called Coverage to evaluate the connection among passwords and individual data. Individual PCFG breaks passwords a lot quicker than PCFG also, makes online assaults significantly more prone to succeed. We look at the utilization of basic twisting capacities that are picked by clients to alleviate undesirable relationship between individual data and passwords. To expand secret phrase security, online verification frameworks have begun to uphold stricter secret phrase approaches. Secret word re-age strategy is accessible in this framework.

\section{III.PROPOSED METHOD}

To ensure passwords in a confirmation information table, the framework planner should initially choose a cryptographic hash work and a symmetric-key calculation, where the condition that should be fulfilled is that the size of the hash estimation of the chose cryptographic hash work is equivalent to the critical size of the chose symmetric-key calculation. For comfort, a few matches of cryptographic hash capacities also, symmetric-key calculations. Moreover, cryptographic hash capacities and symmetric-key calculations that are most certainly not recorded here could likewise be utilized in the ENP, which sufficiently demonstrates the adaptability of our system. The proposed structure depends on the ENP; subsequently, for better understanding, the information stream chart of the age method of the ENP.

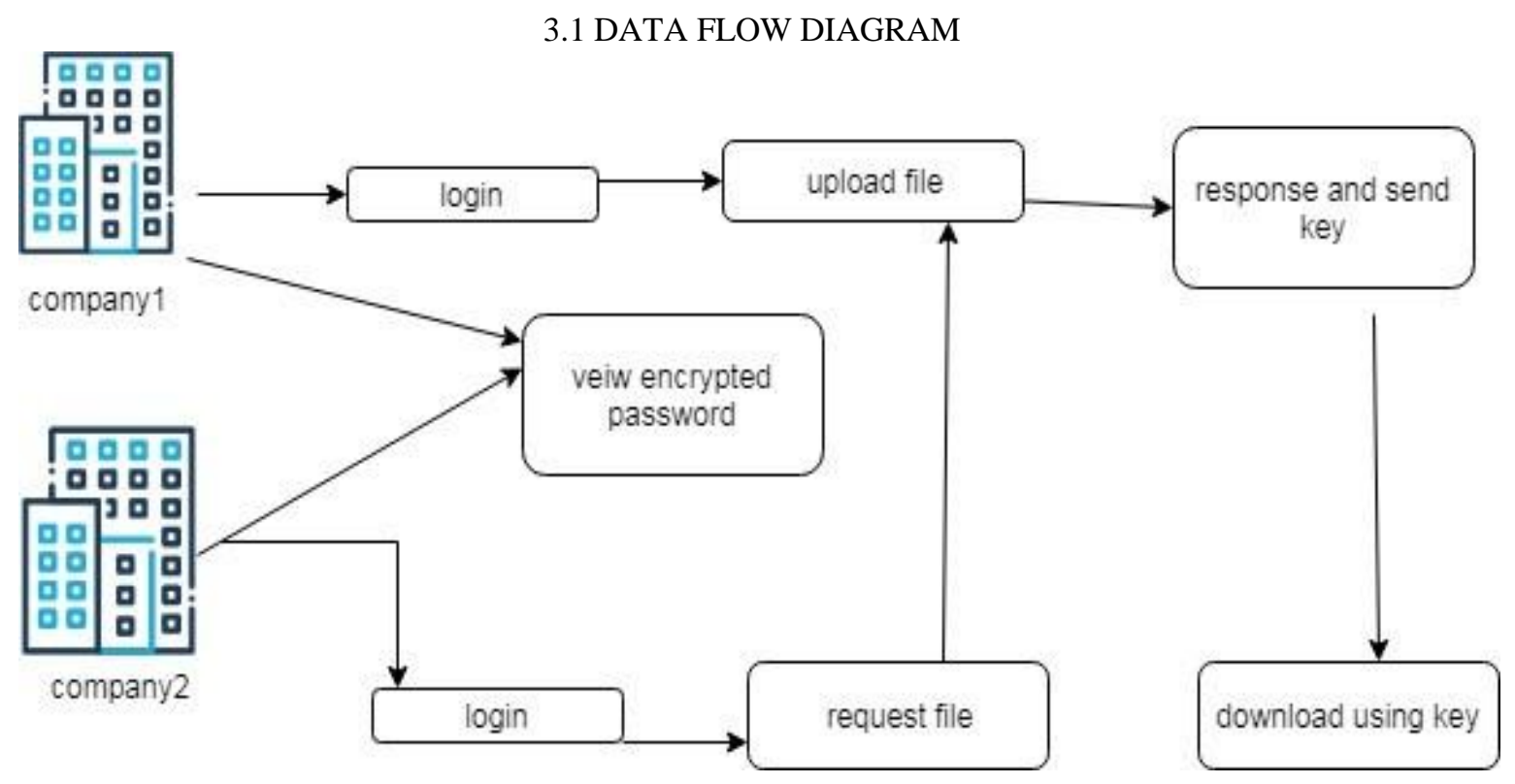




\section{MODULE DESCRIPTION}

\section{USER INTERFACE DESIGN:}

- This is the first module of our project. The important role for the user is to move login window to user window.

- This module has created for the security purpose. In this login page we have to enter login user id and password.

- It will check username and password is match or not (valid user id and valid password).

- If we enter any invalid username or password we can’t enter into login window to user window it will shows error message.

- So we are preventing from unauthorized user entering into the login window to user window.

- It will provide a good security for our project. So server contain user id and password server also check the authentication of the user.

- It well improves the security and preventing from unauthorized user enters into the network.

- In our project we are using JSP for creating design. Here we validate the login user and server authentication.

\section{VIEWS ENCRYPTED PASSWORD.}

- In this part registered user will view the encrypted passwords from the database stored.

- After registration from the each company or users they will view their encrypted passwords.

\section{UPLOAD FILE FROM COMPANY1.}

- In this module, after logging in company have to upload some files i.e. to be pdf or a text file.

- The uploaded file gets encrypted and stored in the database. With that also tha hacker cant able to access files.

\section{COMPANY 2 VIEWS UPLOADED FILE.}

- In another side, company2 login and view the files uploaded by other company.

- They can only view the name of the file. They cannot get or download file directly.

\section{REQUEST FOR SOME FILE.}

- Here Company2 request for some files uploaded by the company1.

- Directly they can click on request button it will be sent to the company1 as a notification.

\section{COMPANY 1 ACCEPTS WITH SENDING KEY.}

- Company1 receives notification after getting log in. here there will be the request sent by other company.

- If they accept means, key will be generated for download the file. The key will be sent to the requested company for downloading file with acceptance notification. Otherwise it will be rejected .

\section{COMPANY 2 DOWNLOADS FILE USING KEY.}

- Here the acceptance notification will be received with the key. When he downloads the file it asks for entering the key.

- If it is matched it will be downloaded. 


\title{
IV. TECHNIQUES AND ALGORITHM
}

\author{
MD5 (hash) ALGORITHM:
}

- MD5 algorithm is an asymmetric cryptography algorithm. Asymmetric means that it works on two different keys i.e. Public Key and Private Key.

- As the name suggests that the Public Key is given to everyone and Private Key is kept private.

- The MD5 work is a cryptographic calculation that takes a contribution of self-assertive length and produces a message digest that is 128 pieces in length.

- The digest is here and there likewise called the "hash" or "finger impression" of the info.

- MD5 is utilized as a rule where a possibly long message should be handled and additionally analyzed rapidly.The most common application is the creation and verification of digital signature.

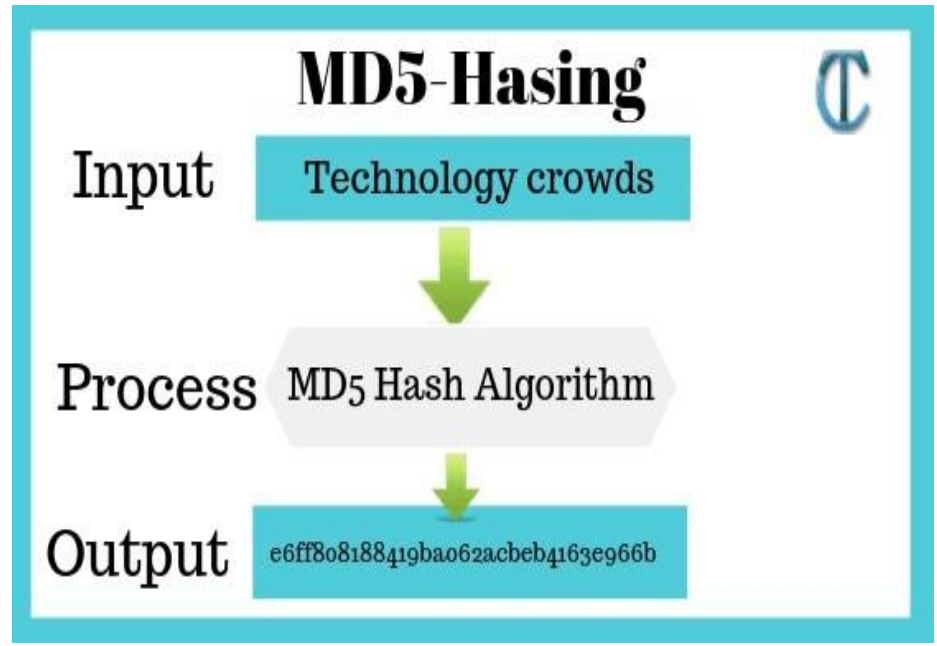

\section{V.CONCLUSIONS}

In this paper, we proposed a mysterious expression security plot called ENP, and presented a mysterious expression affirmation framework reliant on the ENP. In our design, the segments in the affirmation data table are ENPs. Ultimately, we examined and investigated the attack multifaceted design of hashed secret word, salted mystery word, key broadening and the ENP. The results show that the ENP could go against inquiry table attack and give more grounded secret word confirmation under word reference attack. It justifies referring to that the ENP needn't waste time with extra segments (e.g., salt) while restricting question table attack.

\subsection{FUTURE WORK}

Later on, other NDB age figurings will be inspected and familiar with the ENP to also improve secret expression security. Moreover, various methodologies, for instance, complex affirmation and challenge-response affirmation.

\section{REFERENCES}

[1] J. Bonneau, C. Herley, P. C. van Oorschot, and F. Stajano, "Passwords and the evolution of imperfect authentication," Communi cations of the ACM, vol. 58, no. 7, pp. 78-87, Jun. 2015. 
[2] M. A. S. Gokhale and V. S. Waghmare, "The shoulder surfing resistant graphical password authentication technique," Procedia Computer Science, vol. 79, pp. 490-498, 2016.

[3] J. Ma, W. Yang, M. Luo, and N. Li, “A study of probabilistic password models," in Proceedings of 2014 IEEE Symposium on Security and Privacy, May 2014, pp. 689-704.

[4] A. Adams and M. A. Sasse, "Users are not the enemy," Communications of the ACM, vol. 42, no. 12, pp. 40-46, Dec. 1999.

[5] E. H. Spafford, "Opus: Preventing weak password choices," Computers \& Security, vol. 11, no. 3, pp. 273-278, 1992.

[6] Y. Li, H. Wang, and K. Sun, "Personal information in passwords and its security implications," IEEE Transactions on Information Forensics and Security, vol. 12, no. 10, pp. 2320-2333, Oct. 2017.

[7] D. Florencio and C. Herley, "A large-scale study of web password habits," in Proceedings of the 16th International Conference on World Wide Web. ACM, 2007, pp. 657-666.

[8] R. Shay, S. Komanduri, A. L. Durity, P. S. Huh, M. L. Mazurek, S. M. Segreti, B. Ur, L. Bauer, N. Christin, and L. F. Cranor, "Designing password policies for strength and usability," ACM Transactions on Information and System Security, vol. 18, no. 4, pp. 13:1-13:34, May 2016.

[9] D. Wang, D. He, H. Cheng, and P. Wang, "fuzzyPSM: A new password strength meter using fuzzy probabilistic context-free grammars," in Proceedings of 2016 46th Annual IEEE/IFIP International Conference on Dependable Systems and Networks, Jun. 2016, pp. 595-606.

[10] H. M. Sun, Y. H. Chen, and Y. H. Lin, "oPass: A user authentication protocol resistant to password stealing and password reuse attacks," IEEE Transactions on Information Forensics and Security, vol. 7, no. 2, pp. 651-663, Apr. 2012.

[11] M. Zviran and W. J. Haga, "Password security: An empirical study," Journal of Management Information Systems, vol. 15, no. 4, pp. 161- 185, 1999.

[12] P. Andriotis, T. Tryfonas, and G. Oikonomou, "Complexity metrics and user strength perceptions of the pattern-lock graphical authentication method," in Proceedings of Human Aspects of Information Security, Privacy, and Trust. Springer International Publishing, 2014, pp. 115- 126. 